\title{
Bridging the treatment gap: the secondary care perspective
}

\section{Dancy}

Heart 2005;91(Suppl II):ii32-ii34. doi: 10.1136/hrt.2005.062083

Protocols and integrated care pathways can be valuable in the hospital care of patients with heart failure or left ventricular systolic dysfunction after acute myocardial infarction. A designated member of staff, often a specialist nurse, must be responsible for identifying patients suitable for management by the protocol and for ensuring that the protocol is adhered to. A new training scheme for "limited echocardiography" might enable specialist nurses to investigate left ventricular function within the first 24 hours of admission. Patients should be discharged from hospital as soon as they are out of danger. At present, they are often kept in hospital for process reasons. A "continuing care" clinic run by a specialist nurse, where patients can be seen daily after discharge until they are stabilised, is one way of bridging the gap between secondary and primary care. Communication between secondary and primary care needs to improve and same day discharge summaries are essential.

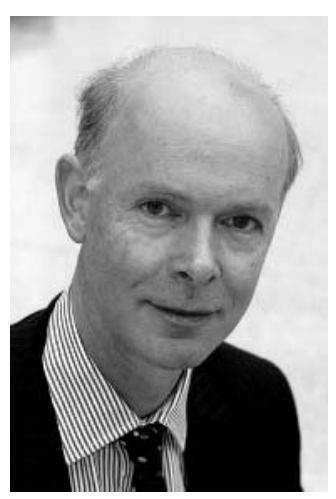

$\mathrm{n}$ a patient with acute myocardial infarction (MI), the management of the MI itself may overshadow the management of heart failure or left ventricular systolic dysfunction (LVSD). There is a tendency for medical staff to concentrate on thrombolysis, rhythm disturbances, revascularisation, and resynchronisation therapy, with lower priority being given to pharmacological treatment of heart failure and LVSD. However, left ventricular function is one of the principal determinants of life expectancy after myocardial infarction and hospitals have an important role to play in establishing management objectives. It is well recognised that patients are more likely to receive the correct treatments long term if they are started in hospital. ${ }^{1}$

The process of ventricular remodelling is thought to start immediately after the infarct and there is therefore good reason to start drugs for LVSD early. Of course, the early introduction of $\beta$ blockers after infarction was recommended as long as 20 years ago by the early ISIS studies. There is also evidence that angiotensin converting enzyme (ACE) inhibitors should be introduced within the first 24 hours of MI if possible: the GISSI-3 trial $^{2}$ showed that starting an ACE inhibitor (lisinopril) within 24 hours of symptom onset reduced mortality. Treatment for six weeks produced benefit at six months, with echocardiographic evidence of less remodelling. However, introduction of these drugs needs to be undertaken with some caution in view of the potential risks involved with treating previously hypertensive patients who have low blood pressure $(<120 \mathrm{~mm} \mathrm{Hg})$ on admission.

\section{LOGISTICS OF MANAGEMENT OF HEART FAILURE}

In the post-MI situation, the management of heart failure and LVSD is only part of the challenge. There are logistic issues to consider. For example, patients may be on a variety of wards, and they are often moved within the hospital during their stay. Many patients are not on the coronary care unit, and they may not be under the care of a cardiologist. In this situation, protocols and integrated care pathways can be valuable. Many hospitals now have both acute $\mathrm{MI} /$ chest pain protocols and acute heart failure protocols. It may be preferable for instructions for treating LVSD in the context of AMI to be incorporated into the AMI/chest pain protocol rather than the acute heart failure protocol, since it can be confusing if there are two protocols being implemented for the same patient.

The advantages of protocols are that they:

- provide the potential for standardised care, even if staff and location of patients change

- provide a "check list" to reduce the risk of tasks being forgotten

- increase the chances of the correct decisions being made.

However, protocols do have limitations. The patient has to be identified as suitable for a particular protocol, and the protocol has to be followed: the fact that the patient is being managed "per protocol" does not necessarily mean that the protocol is being adhered to. There can be problems with "unusual" patients who do not appear to fit the protocol, and some health professionals are concerned that use of a protocol will limit the scope for lateral thinking, particularly when the patient has co-morbidity.

\section{IDENTIFICATION OF PATIENTS FOR PROTOCOL BASED CARE}

All patients who come into hospital with an MI need to be identified and managed by the protocol. However, it is not sufficient to design a protocol and place it on the wards. A designated member of staff must be responsible for overseeing the process and for ensuring that

Abbreviations: $A C E$, angiotensin converting enzyme; GISSI, gruppo Italiano per lo studio della sopravvivenza nell' infarto miocardio; GP, general practitioner; ISIS, international study of infarct survival; LVSD, left ventricular systolic dysfunction; MI, myocardial infarction 
all patients are managed by the protocol. This responsibility is often given to a specialist nurse.

The role of specialist nurses differs according to local practice. Some hospitals have heart failure nurses and some have chest pain nurses. One of the more difficult aspects of the specialist nurse role is that if patients remain under the admitting team, as is the case in some hospitals, the nurse will need to work with different teams. These teams may be more or less receptive to the nurse, who needs to know how much he or she is allowed to do. There is no doubt that the best system of management is for all patients to be admitted under a cardiologist and to stay on a cardiological ward. In this situation, the nurse knows exactly who he or she is working with and what is expected of him or her.

If heart failure treatments are to be introduced within the first 24 hours, echocardiography also needs to be available within that time frame. This can be difficult to achieve as echocardiography services are stretched in most cardiology departments. The availability of portable echocardiography machines on the coronary care unit can help, but there might still be a problem in some hospitals because of difficulty in recruiting suitably trained technicians. The British Society for Echocardiography is currently developing a training scheme for "limited echocardiography" to investigate left ventricular function. In the future, specialist nurses might undertake this training so that they can carry out echocardiography in the first 24 hours and provide the data to inform the patient's subsequent treatment.

\section{PATIENT DISCHARGE AFTER ACUTE MI}

There is an unwritten rule in many hospitals that a patient with an MI should stay in hospital for five nights. However, in line with a general trend towards early discharge, many centres, particularly in the USA, are moving towards early discharge after MI if the patient is stable. The use of primary angioplasty is associated with improved reperfusion and potentially better outcomes following MI. ${ }^{3}$ The UK government is funding a pilot project to study the feasibility of introducing a national primary angioplasty service. ${ }^{4}$ However, unless such a service were to be centrally funded, it is likely that purchasers would only fund it if they could be persuaded that it would result in earlier discharge; in other words, that a reduction in length of stay would fund the service development.

Patients need to stay in hospital if they have continuing ischaemia, unstable cardiac rhythms, uncontrolled heart failure (pulmonary oedema or a continued need for intravenous medication) or if they require further procedures. However, at present patients are often kept in hospital for "process" reasons, such as for making minor adjustments to medication, for weighing, for blood tests, and for

\section{Box 1: Post-discharge activities}

- Further investigations

- Titrating up drugs that have been introduced

- Monitoring progress

- Assessing response to treatments

- Keeping the patients abreast of their condition

- General patient education

- Rehabilitation

- Secondary prevention and lifestyle changes (for example, smoking)

- Chronic disease management
Learning points

- Systematic care is needed if heart failure management is to be optimised

- Systematic care requires sound clinical protocols and well trained people (often specialised nurses) to ensure that the protocols are put into action

- Early discharge is possible provided that daily follow up can be arranged until the patient's condition is stabilised

- Good communication between secondary and primary care is essential

echocardiography, all of which could be done on an outpatient basis if the supporting processes were available.

When a post-MI patient is discharged there are still a number of procedures to be carried out (box 1). While further investigations are definite secondary care type activities and secondary prevention, lifestyle change and chronic disease management are definite primary care type activities, the other tasks listed could all be done by any health professional with the appropriate skills in any setting. This provides an opportunity for alternative arrangements to be made.

The traditional model for transition from secondary to primary care is that a discharged patient is looked after in primary care, and given a six week outpatient appointment. However, primary care often has insufficient evidence on which to base any decisions that might need to be taken within that six week period. Consequently, with this traditional model there is a reluctance to discharge patients who are relatively stable but still need attention, such as for adjustment of their drug treatment.

With the move towards earlier discharge, many tasks that traditionally would be carried out in the hospital will now need to be done on an outpatient basis. In this respect, there is a case to be made for "continuing care" clinics, run within secondary care, with the potential to see patients daily after their discharge, if necessary, for continued uptitrating of drugs, blood tests, and so on. The availability of such clinics would mean that it is possible to discharge the patient while keeping a close eye on them. The system would be resource intensive and would need to be financed appropriately, but it could answer some of the problems associated with the move towards early discharge that purchasers are encouraging.

\section{THE DISCHARGE PROCESS}

More effort needs to be taken to achieve good communication between secondary and primary care. Various initiatives should help with this, including the general practitioner (GP) with a specialist interest scheme and the national programme for information technology. It is also important to achieve same day discharge summaries. Electronic discharge summaries are increasingly being used and these give the opportunity to suggest appropriate Read codes for primary care, to ensure that the patient is entered on to the practice disease register. The discharge summary must contain future management principles, which may involve joint protocols. The GP needs to know why the patient is taking a particular drug. For example, information on whether the patient is taking an ACE inhibitor for hypertension or for heart failure/ LVSD will influence the GP's response if the patient develops side effects from their drug treatment. Details of follow up arrangements (continuing care and planned investigations) must also be included on the summary. 


\section{CONCLUSION}

Left ventricular function is a key determinant of life expectancy after MI. The management of LVSD and heart failure should be incorporated into $\mathrm{MI} /$ chest pain protocols and there needs to be a designated individual—or more than one individual-who is responsible for identifying patients with MI and ensuring that they are managed by the protocol. Echocardiography needs to be available on day 1, so that the appropriate treatments can be introduced with as much background knowledge about the extent of the infarct as possible.

Patients should be discharged from hospital as soon as they are out of danger. There then needs to be a system for seeing patients daily after discharge to manage the process until the patient is stabilised. A "half way house" clinic, run by a nurse specialist, for patients who have recently been discharged is one way of bridging the gap between secondary and primary care. Following stabilisation, chronic disease management should be part of primary care operations.

\section{REFERENCES}

1 Fonarow GC. Strategies to improve the use of evidence-based heart failure therapies: OPTIMIZE-HF. Rev Cardiovasc Med 2004:5(suppl 1):S45-54.

2 GISSI-3 Investigators. Gruppo Italiano per lo studio della sopravivenza nell'infarto miocardio. GISSI-3: effect of lisinopril and transdermal glyceryl trinitrate single and together on 6-week mortality and ventricular functionafter acute myocardial infarction, Lancet 1994;343:1115-22.

3 Keeley EC, Boura JA, Grines CL. Primary angioplasty versus intravenous thrombolytic therapy for acute myocardial infarction: a quantitative review of 23 randomised trials. Lancet 2003;361:13-20.

4 Department of Health. The national service framework for coronary heart disease. Winning the war on heart disease. Progress report 2004. London: Department of Health, 2004. 